\title{
VENUS EXOSPHERIC STRUCTURE: THE ROLE OF SOLAR RADIATION PRESSURE
}

\author{
JAMES BISHOP \\ Department of Atmospheric, Oceanic and Space Sciences, University of Michigan, \\ Space Research Building, Ann Arbor, MI 48109-2143, U.S.A.
}

(Received 4 January 1989)

\begin{abstract}
The existence of a "hot" population of hydrogen atoms in the Venus exosphere is well known. In the outer coronal region where it is dominant $\left(r \geqslant 2.0 R_{\mathrm{V}}\right)$, hydrogen atoms are also subject to a relatively strong radiation pressure exerted by resonant scattering of solar Lyman- $\alpha$ photons. Collisionless models illustrating the consequent structure are discussed, with the nonthermal population mimicked by a dual Maxwellian exobase kinetic distribution. In these models, a considerable fraction of the "hot" atoms outside $2.0 R_{\mathrm{V}}$ belongs to the quasi-satellite component, this fraction exceeding $1 / 2$ for $4.0 R_{\mathrm{V}} \leqslant r \leqslant 10.0 R_{\mathrm{V}}$. Quasi-satellites also raise the kinetic temperature near $2.0 R_{\mathrm{V}}$ by $\sim 150 \mathrm{~K}$. Solar ionization of bound atoms occurs mainly outside the ionopause, yielding a partial escape flux $\geqslant 2 \times 10^{6} \mathrm{~cm}^{-2} \mathrm{~s}^{-1}$ over the dayside exobase for assumed solar conditions. The inclusion of a cold exobase prescribed by Pioneer Venus observations has little influence on the outer region (in particular, the quasi-satellite component is unaltered) except that the transition to "hot" kinetic character occurs closer to the exobase on the nightside due to the colder main exobase temperatures there. Lastly, a "tail" of bound atoms is formed as in the terrestrial situation.
\end{abstract}

\section{INTRODUCTION}

The hydrogen atom exosphere of Venus has been of considerable interest primarily because of its "dual" appearance, seeming to have two temperatures, and the implications this carries for loss of hydrogen over geologic time scales. Measurements obtained with photometers on board various flyby missions have come to be analyzed in terms of a dual exosphere (Anderson, 1976; Takacs et al., 1980; Bertaux et al., 1982) comprised of a "hot" atom corona superimposed onto a true thermal exosphere of the sort presented by Chamberlain (1963). Considerable work has gone into elucidating the major sources of the nonthermal population: charge exchange collisions with ionospheric protons and singly ionized oxygen (Chamberlain, 1977; Cravens et al., 1980; Hodges and Tinsley, 1981, 1986: Kumar et al., 1983; Rodriguez et al., 1984), momentum transfer collisions with other "hot" constituents (mainly atomic oxygen) (McElroy et al., 1982; Kumar et al., 1983 ; Rodriguez et al., 1984), and charge exchange collisions with solar wind protons (Bertaux et al., 1978). Most often, however, in the reduction and subsequent interpretation of photometer measurements the nonthermal population has simply been modeled as a separate classical exosphere of higher exobase temperature and smaller exobase density. This picture of a dual exobase is not implausible in view of the limited region within which ionospheric charge exchange collisions occur, in that these are contained by the low-lying ionopause. This is in contrast with the situation on Earth, where charge exchange collisions occur throughout the voluminous plasmasphere and no molecular ions survive to exobase altitudes.

Another distinction with the geocoronal situation is a relatively stronger acceleration imposed on exospheric hydrogen via resonant scattering of solar Lyman- $\alpha$ photons, since Venus is closer to the Sun. Radiation pressure acts to modify the trajectories executed by exospheric atoms. This can lead to structural features that are to a large extent independent of whatever sources and sinks of constituent atoms may be active. An example of such a feature is the geotail phenomenon first observed with photometers on board $O G O-5$ (Thomas and Bohlin. 1972: Bertaux and Blamont, 1973) and more clearly seen with the ultraviolet imaging photometer on $D E-1$ (Rairden $e t$ al., 1986).

In this paper, the intention is to investigate effects related to radiation pressure. The concern is with "evaporative" models, with the nonthermal population mimicked by a hot exobase source. Since several factors governing the actual Venus cxosphere are ignored (e.g., thermospheric winds and variations in ionospheric quantities), these models cannot be claimed to be "realistic". Rather, as in the parallel study of the geocorona (Bishop, 1985; Bishop and 
Chamberlain, 1987), the intent is to analyze the physics of the Venus exosphere in a systematic fashion. Solar ionization and exobase nonuniformities are easily retained in a collisionless formulation; in view of the dramatic diurnal contrast in exobase temperature (Niemann et al., 1980) and atomic hydrogen density (Brinton et al., 1980) on Venus, such retention is necessary even in a "first order" treatment. Accordingly, the cases considered in this paper extend to exospheres arising from exobase models based on in situ Pioneer Venus measurements and subject to solar ionization. Attention is concentrated on locations well above the ionopause.

\section{EXOSPHERIC SETTING AT VENUS}

As a point of definition, collisions in an exosphere are infrequent enough that particle trajectories must be explicitly taken into account; these are defined by the acting forces and can in general be grouped into physically distinct classes, leading to the idea of exospheric components. Evaporative models are constructed by taking the exospheric kinetic distribution $f$ (i.e., the number of atoms in an element of volume dr about a position $\mathbf{r}$ with velocities in a range $d \mathbf{v}$ about a value $v$ ) to be entirely determined by an assumed kinetic distribution at the exobase $f_{\mathrm{c}}$ without intervening collisions, with no infall of atoms from interplanetary space (i.e., there are no "hyperbolic" or capture components). It is convenient and often very useful to suppose an isotropic Maxwellian for $f_{\mathrm{c}}$ characterized by a uniform temperature and constituent density over the (spherical) exobase (Chamberlain, 1963). Variations in the kinetic distribution across an exosphere of this sort are then due solely to gradients in potential energy, while variations in derived quantities like number density or kinetic temperature (i.e., moments of $f$ ) also reflect variations in the volume of velocity space associated with each trajectory class. The ideal collisionless approach is thus an appropriate starting point in that it reveals the structure imposed by the force fields.

Radiation pressure is treated in this work as a uniform antisolar acceleration $a$, represented by a potential mar $\cos \chi$ superposed on the planetary gravitational potential $G M m / r$; here $G$ is the gravitational constant, $M$ the mass of the planet, $m$ the hydrogen atom mass, $r$ the planetocentric distance, and $\chi$ the solar angle. It arises via the resonant scattering of solar Lyman- $\alpha$ photons, which impart on the average a net momentum impulse $h v / c$ per photon in the antisolar direction, where $h$ is the Planck constant, $c$ the speed of light, and $v$ the photon frequency. The radiation pressure acceleration is then given by $a=g(1216 \mathrm{~A}) h v / m c$, where $g(1216 \mathrm{~A})$ is the number of solar Lyman- $\alpha$ photons scattered per second by a hydrogen atom in the neighborhood of Venus. In the trajectory calculations used to construct the models presented in this paper, a solar flux at Lyman- $\alpha$ line center of $4.2 \times 10^{11}$ photons $\mathrm{cm}^{-2} \mathrm{~s}^{-1} \mathrm{~A}^{-1}$ at 1.0 a.u. was assumed, yielding an acceleration $1.44 \mathrm{~cm} \mathrm{~s}^{-2}$ at Venus. The exopause, the planetocentric distance $r_{\mathrm{p}}$ beyond which the radiation pressure acceleration exceeds the planetary gravitational acceleration, is consequently at $24.8 R_{\mathrm{V}}$. An atom of sufficient energy to climb beyond this radius is considered to have escaped the planet altogether.

The trajectories defined in these superposed force fields can depart significantly from the Keplerian orbits usually pictured in exospheric modeling (Bishop and Chamberlain, 1989). In particular, the introduction of radiation pressure eliminates the notion of atoms executing closed orbits. One can continue to discuss trajectories in terms of Keplerian orbital elements, although one must then recognize that these quantities will evolve. This has been discussed in the tightly bound case by Chamberlain (1979), who showed that the primary effect of resonant photon scattering is then an evolution of orbital angular momentum (or eccentricity) while the energy remains effectively constant. In general, "satellite" orbits intersect the exobase and are populated by atoms evaporating out of the collisionally dominated thermosphere in the same way as ballistic trajectories. In the Venus case, the comparatively cold exobase and these trajectory modifications conspire to ensure the presence of an effectively complete thermal satellite component near the planet, to revert to the language of Chamberlain (1963). Another modification is the imposition of an exopause, introduced above. This enhances the escape flux in that escaping atoms no longer need reach infinity. At the cold temperatures of the main exobase, though, the exopause is not an important consideration.

\section{Exobase kinetic distribution}

A dual exobase kinetic distribution of the form

$$
f_{\mathrm{c}}=\frac{N_{\mathrm{c}}}{\left(\pi \rho_{\mathrm{c}}\right)^{3 / 2}} \exp \left[-\psi^{2} / \rho_{\mathrm{c}}\right]+\frac{N_{\mathrm{h}}}{\left(\pi \rho_{\mathrm{h}}\right)^{3 / 2}} \exp \left[-\psi^{2} / \rho_{\mathrm{h}}\right]
$$

has been used, where $\rho_{\mathrm{c}}=T_{\mathrm{c}} / T_{\text {ref }}, \rho_{\mathrm{h}}=T_{\mathrm{h}} / T_{\text {ref }}$ and $\psi=v / U$ is the dimensionless speed, $U=\left(2 k T_{\mathrm{ref}} / m\right)^{1 / 2}$ being the most probable speed of a hydrogen atom 
at the temperature $T_{\text {ref }}$ ( $k$ denotes the Boltzmann constant). The first term is simply the cold Maxwellian, with the exobase temperature $T_{\mathrm{c}}$ and atomic hydrogen density $N_{\mathrm{c}}$ specified by model. For those cases involving a uniform exobase, $T_{\mathrm{c}}$ has the reference value $T_{\text {ref }}=312 \mathrm{~K}\left(\rho_{\mathrm{c}}=1\right)$ and $N_{\mathrm{c}}=10^{5} \mathrm{~cm}^{-3}$. The second term is meant to mimic in a very simple way the nonthermal or "hot" population revealed by observations; this population dominates the Venus exosphere at radii greater than $2.0 R_{\mathrm{V}}$. Again, while the mechanisms involved in the generation of "hot" atoms and their variation over the history of the planet pose important questions, they are simply sidestepped in this paper through the use of the two temperature exobase. Throughout, $N_{\mathrm{h}}$ and $T_{\mathrm{h}}$ have the values $10^{3} \mathrm{~cm}^{-3}$ and $1000 \mathrm{~K}$, respectively. The exobase radius has been taken to be $6305 \mathrm{~km}$.

\section{Exobase nonuniformities}

The variation in main exobase temperature with solar angle $\chi$ is fairly well known as a result of the Pioneer Venus mission. Hedin et al. (1983) outline an empirical modeling scheme for thermospheric quantities wherein a fifth-order spherical harmonic expansion was fitted to a subset of in situ measurements (primarily ONMS data) ; the relation for exobase temperature prescribed in this way [refer to Hedin et al., 1983, equations (A6), (A23)-(A26) and Table 1] has been used to generate $\rho_{\mathrm{c}}$ for the nonuniform evaporative cases discussed below. It is necessary to specify the level of solar activity in using this relation. For the adopted value of the radiation pressure acceleration, an $F_{10.7}$ value of 220 is indicated by Fig. 7 of
Paxton et al. (1988); the resulting exobase temperature is illustrated in Fig. 1 for ecliptic plane locations. Also shown are the corresponding atomic hydrogen densities $N_{\mathrm{c}}$ given by an interpolation scheme based on the charge-exchange equilibrium results of Brinton et al. (1980, Fig. 3), extrapolated to an exobase altitude of $250 \mathrm{~km}$ assuming diffusive equilibrium at the local exobase temperature. Although the true exobase altitude varies with solar angle and is generally below $250 \mathrm{~km}$, little error can arise by reference to a fixed altitude, provided it is low enough that the kinetic distribution remains Maxwellian and controlled by local conditions.

The absence of a deflecting magnetosphere and the relative nearness to the Sun imply a greater significance for solar ionization processes for the Venus exosphcre than at Earth; a solar ionization (SI) decay time of $7.6 \times 10^{5} \mathrm{~s}$ has been adopted, encompassing both photoionization (under moderate solar conditions) and charge exchange collisions with an unattenuated solar wind (Hodges and Tinsley, 1981).

\section{Calculation scheme}

The procedure used here to construct an exosphere model starts with the selection of the locations at which quantities like density or kinetic temperature are to be evaluated. As in the geocoronal studies, these locations have been chosen as being aligned along the planet-Sun axis in the solar $\left(\chi=0^{\circ}\right)$ and antisolar $\left(\chi=180^{\circ}\right)$ directions, with the radial distances specified by the numerical technique used to obtain the radial column density between the exobase and exopause. Actually, it is more convenient to work in

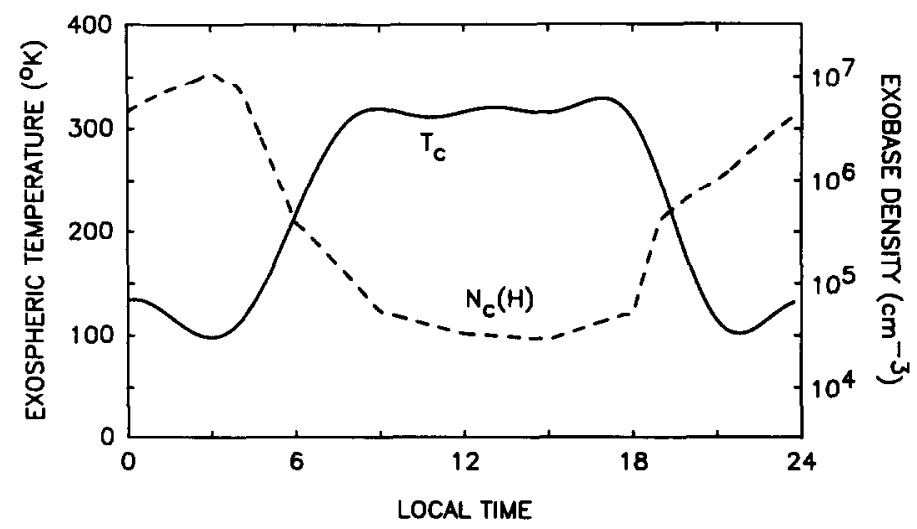

Fig. 1. EXOBASE TEMPERATURE $T_{\mathrm{c}}$ FROM THE HEDIN et al. (1983) MODEL AND ATOMIC HYDROGEN DENSITY $N_{\mathrm{c}}$ BASED ON THE RESULTS OF BRINTON et al. (1980) AT ECLIPTIC PLANE LOCATIONS AND AN EXOBASE ALTITUDE OF $250 \mathrm{~km}$.

The pre-dawn bulge in $N_{\mathrm{c}}$ has been centered on the ecliptic plane. 
terms of the dimensionless gravitational potential $\lambda=G M m / k T_{\text {ref }} r$, in terms of which the column density can be written as

$$
N_{\text {col }}(\lambda, \chi)=\frac{G M m}{k T_{\text {ref }}} \int_{\lambda_{p}}^{\lambda} N\left(\lambda^{\prime}, \chi\right) / \lambda^{\prime 2} \mathrm{~d} \lambda^{\prime}
$$

where $N\left(\lambda^{\prime}, \chi\right)$ is the local number density. A 16-point Gauss-Legendre quadrature over $\lambda$ has been used in evaluating $N_{\text {col }}$. Likewise, the velocity space integration

$$
N(\lambda, \chi)=\iiint f(\lambda, \chi, v) \mathrm{d}^{3} v
$$

is reliably carried out using Gauss-Legendre quadratures. Note that the trajectories encompassed by this integral remain planar. Recasting in terms of the dimensionless speed $\psi=v / U$,

$$
N(\lambda, \chi)=\iiint^{\prime} f(\lambda, \chi, \psi, \mu, \varepsilon) \psi^{2} \mathrm{~d} \psi \mathrm{d} \mu \mathrm{d} \varepsilon
$$

where $f$ has the normalization indicated in equation (1) and where $\mu=\cos \delta, \delta$ being the inclination of the velocity vector with respect to local zenith and $\varepsilon$ the angle between the plane of the ecliptic and the plane of motion. In some of the models discussed in this paper, the planet-Sun axis is an axis of symmetry, in which case the $\varepsilon$-integral reduces to a factor of $2 \pi$.

(Refer to Bishop, 1985, Fig. 1, for an illustration of the coordinate scheme.)

In evaluating the integrals, it is necessary to recognize the existence of distinct regions in velocity space, over which the kinetic distribution varies smoothly but between which can vary abruptly or even discontinuously. One of the distinguishing features of an cxospheric kinetic distribution is its lack of isotropy; the absence of atoms moving downward $(\mu<0)$ with speeds in excess of the local escape speed is the simplest example of this. Thus prior to converting the restricted integral in equation (4) to a quadrature summation, it is broken into component contributions along the lines laid out in Chamberlain (1963). A complication arises in that due to the action of radiation pressure, the integration limits are not immediately available in analytic expressions but can be determined numerically; this has been done by iterative scarches using the limits specified by the Chamberlain theory as starting points. Once the limits are known, the quadrature formulae specify those values of $\psi$ and $\mu$ for which the kinetic distribution $f$ must be evaluated. The trajectories so specified are propagated using the equations of motion

$$
\begin{aligned}
& \frac{\mathrm{d} \lambda}{\mathrm{d} \tau}=-\lambda^{2} \psi \cos \delta \\
& \frac{\mathrm{d} \chi}{\mathrm{d} \tau}=\lambda \psi \sin \delta \\
& \frac{\mathrm{d} \psi}{\mathrm{d} \tau}=\frac{-\lambda^{2} \cos \delta}{2}-\alpha \cos (\delta+\chi) \\
& \frac{\mathrm{d} \delta}{\mathrm{d} \tau}=\frac{\lambda}{\psi} \sin \delta\left(\frac{\lambda}{2}-\psi^{2}\right)+\frac{\alpha}{\psi} \sin (\delta+\chi)
\end{aligned}
$$

where $\tau$ is the dimensionless time $\left(\tau=\left(2^{1 / 2} / G M\right)\right.$ $\left.\left(k T_{\text {ree }} / m\right)^{3 / 2} t\right), \alpha$ the dimensionless radiation pressure acceleration $\left(\alpha=(G M / 2)\left(m / k T_{\text {ref }}\right)^{2} a\right)$, and the remaining variables have previously been defined. After determining the points of intersection of a trajectory with the bounding surfaces (i.e., the exobase or exopause), the kinetic distribution is obtained in the general case by integrating Boltzmann's equation along the specified trajectory, while arbitrary exobase conditions can be imposed. In the simple models used here, collisions of all sorts are dispensed with, allowing the exospheric kinetic distribution to be written down immediately by invoking Liouville's theorem. Solar ionization alters the kinetic distribution according to a factor $\exp \left(-\tau_{\mathrm{f}} / \tau_{\mathrm{s}}\right)$ where $\tau_{\mathrm{s}}$ is the net decay time for either photoionization or charge exchange with fast solar wind protons to occur and $\tau_{\mathrm{f}}$ is the time of flight between the point of launch from the exobase and the exospheric location of interest. The expressions defining the density components, escape flux, and kinetic temperature are given in equations (19)-(21) of Bishop (1985), where the integration limits are more thoroughly discussed.

There are several reasons for isolating and identifying the quasi-satellite component, even though the Keplerian classification of trajectories is not physically appropriatc. It can be uscful as a mcasurc of the extent to which radiation pressure acts to increase the content of a planetary exosphere, as well as to emphasize the modifications to the kinetic distribution caused by radiation pressure dynamics (mainly a partial elimination of the anisotropy of the bound component). Also, a recurring question in the study of the terrestrial exosphere has been the extent of the satellite component as gauged in terms of the satellite critical radius defined in Chamberlain (1963) (see, e.g., Bertaux, 1978; Rairden et al., 1986). A calculation of osculating satellite densities can then facilitate comparisons with estimates derived from measurements (and with results obtained by Monte Carlo simulations, also conventionally analyzed using Keplerian concepts). 


\section{EVAPORATIVE EXOSPHERE MODELS}

\section{Uniform exobase results}

Exospheric quantities corresponding to exobase evaporation with the uniform dual kinetic distribution of equation (1) into the superposed force fields of planetary gravity and solar radiation pressure are displayed in Figs $2-5$. The variation of density along the subsolar $\left(\chi=0^{\circ}\right)$ and antisolar $\left(\chi=180^{\circ}\right)$ axes is shown in Fig. 2 relative to an analytic dual exosphere incorporating an exopause but with satellite atoms suppressed (curve $N_{n}$ of Fig. A.1). The reason for selecting this format is to show more clearly differences between noon and midnight orientations and the analytic models. It also highlights the increase in exospheric content at outer coronal locations due to the generation of an evaporative satcllite component by radiation pressure dynamics; relevant column densities are given in Table 1. The dominance of the hotter component of a dual exosphere at high altitudes ( $r>2.0 R_{\mathrm{V}}$ in this case) is well known and is illustrated in Fig. A.1 (see, e.g., Chamberlain and Hunten, 1987, Section 7.3). The greater enhancement of density along the antisolar axis is a feature attributable to radiation pressure; this is isolated in Fig. 3, along with the "tail" ratio for the cold evaporative population taken alone and the ratio of velocity space volumes at bound component energies (refer to Bishop and Chamberlain, 1987, equation (2) and Fig. 1). In general, the velocity space volume corresponding to the bound component is larger along the midnight axis because of the increased escape speeds there (Bishop and Chamberlain, 1989). In this Venus example, the cold exospheric tail is further augmented by radiation pressure in that an atom launching from the exobase requires a smaller velocity to reach the nightside outer exosphere so that the exobase Boltzmann factor $\exp \left[-m v^{2} / k T\right]$ comes into play. The dual tail is not as pronounced since the hot exobase Boltzmann factor of equation (1) is comparatively immune to energy variations of the order mar $_{\mathrm{c}}$.

The dual quasi-satellite component is effectively all "hot", as illustrated in Fig. 4 along the noon axis; cold population atoms are simply too few in number to contribute at those radii where quasi-satellite trajectories comprise an appreciable fraction of velocity space. Even so, the cold satellite component reveals an interesting variation with radius. At the speeds representative of this component, the cone of acceptance $\delta_{0}\left(\lambda_{c}, \chi_{c}, \varepsilon_{c}\right)$ at the exobase is very flat (defined as the limiting momentum zenith angle separating "hallistic" and "satellite" trajectory behavior: $\delta_{0}\left(\lambda_{c}\right) \leqslant 90^{\circ}$ with equality holding when radiation pressure is absent). Thus, the cold population remains

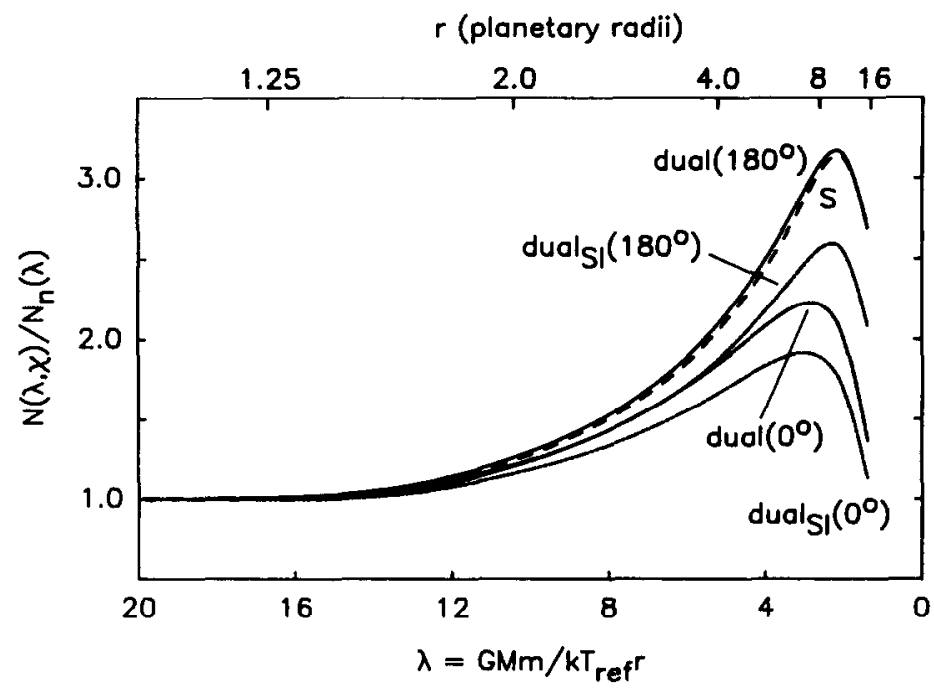

FIG. 2. VARIATION OF EXOSPIIERIC DENSITY WITH DIMENSIONLESS GRAVITATIONAL POTENTIAL $\lambda$ FOR EVAPORATION FROM A UNIFORM DUAL EXOBASE, NORMALIZED POINT BY POINT WITH THE NO-SATELLITE MODIFIED ChamberLaIN MODEL DENSITY $N_{n}(\lambda)$ of FIG. A. 1 .

The resultant profiles along the noon and midnight axes are displayed separately along with the SI-eroded results. Curve $\mathrm{S}$ (dashed) represents the modified Chamberlain model with a complete satellite component $\left(N_{\mathrm{s}}\right.$ of Fig. A.1). 


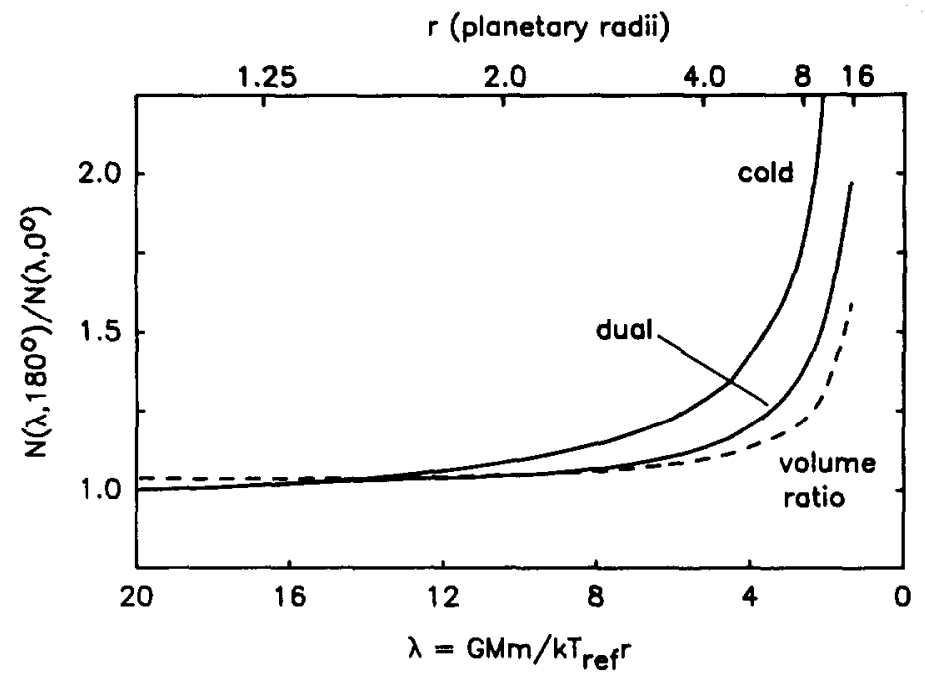

Fig. 3. ExosPheric TAIL RATIOS.

The ratio of density along the midnight axis to equiradial noon axis values is shown for the cold evaporative population and the dual population (uniform exobase conditions). The ratio of velocity space volume for the bound component is also shown, indicating that this is the foundation for the tail phenomenon. The tail ratio is little modified by SI-loss (not shown).

overwhelmingly ballistic at inner coronal locations $\left(r<2.0 R_{\mathrm{V}}\right)$ even though the velocity space volume corresponding to quasi-satellite motions does not remain negligible. It is only at the higher speeds required to attain locations farther out in the exo- sphere that the cone of acceptance at the exobase narrows, forcing the cold satellite fraction to climb to the values set by volume ratios in velocity space (refer to Fig. A.2), as shown in Fig. 4. Dual satellite fractions along the midnight axis (not shown) are generally

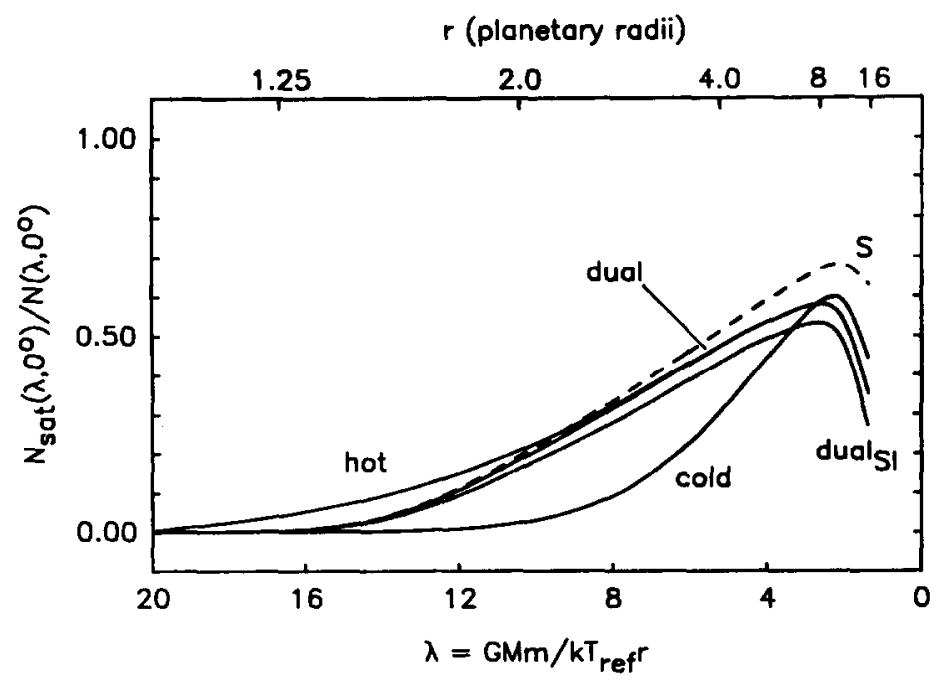

Fig. 4. Satellite fractional densities.

The fractional osculating satellite density profile for the dual evaporative exosphere (uniform exobase conditions) along the noon axis is shown along with the thermal and "hot" component fractions taken alone. Curve $\mathbf{S}$ is the satellite fraction for the dual modified Chamberlain model (from Fig. A.2). Also shown is the SI-modified dual fraction profile for the noon axis. 


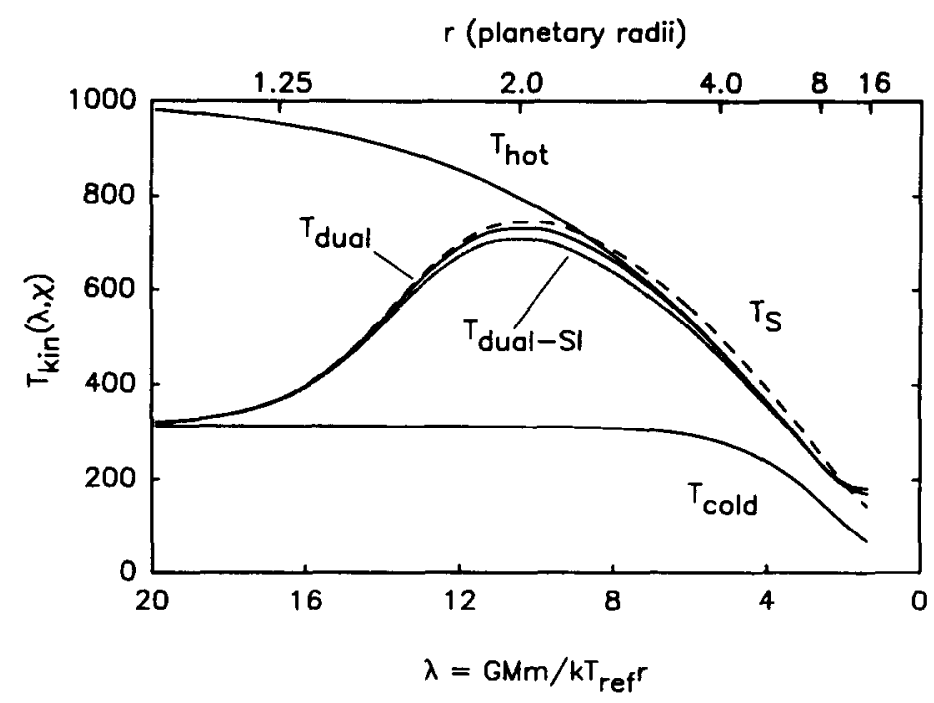

Fig. 5. KinetiC TEMPERATURES.

The kinetic temperatures of the thermal and "hot" evaporative exospheric populations (uniform exobase conditions) along the noon axis are shown. The resultant temperature $T_{\text {dual }}$ shows the dramatic transition in kinetic character occurring near $2.00 R_{\mathrm{V}}$. The temperature given by equation (A.4) is also shown ( $T_{\mathrm{S}}$ of

Fig. A.3), as is the dual SI profile.

larger than equiradial noon axis values, though not greatly so; representative values for the dual model are $0.146(0.139), 0.460(0.428)$, and $0.634(0.568)$ for the midnight (noon) fractions at $1.84 R_{\mathrm{V}}, 3.45 R_{\mathrm{V}}$, and $6.55 R_{\mathrm{V}}$, respectively. This is due to the variation of escape speed with direction of motion in an exosphere subject to radiation pressure: for midnight axis locations, transverse motions generally have higher

TABLE 1. Radial COLUMN DENSITIEs* ABOVE EXOBASE

\begin{tabular}{lcc}
\hline Model & Noon & Midnight \\
\hline Cold Evap. & 3.518 & 3.538 \\
& $(3.516) \dagger$ & $(3.536)$ \\
Dual Evap. & 3.676 & 3.701 \\
& $(3.671)$ & $(3.696)$ \\
HB & 1.190 & 63.69 \\
& $(1.189)$ & $(63.67)$ \\
Dual HB & 1.348 & 63.85 \\
& $(1.344)$ & $(63.83)$ \\
Chamberlain & \multicolumn{2}{c}{3.528} \\
Cold w/o satellites & \multicolumn{2}{c}{3.690} \\
Dual w/ satellites & 1.194 & 63.59 \\
HB (Keplerian) & \multicolumn{2}{c}{}
\end{tabular}

\footnotetext{
* In units of $10^{12} \mathrm{~cm}^{-2}$. Integration of equation (2) extends out to the exopause radius of $24.8 R_{\mathrm{v}}$.

$\dagger$ SI-values appear in parentheses.
}

escape speeds than radial motions, leading to a greater quasi-satellite fractional volume (Bishop and Chamberlain, 1989).

In a dual exosphere model, the fall-off in colder population density with radius causes a countervariation in exospheric kinetic temperature $T_{\text {kin }}$. For Venus, the resulting profile is striking, here illustrated in Fig. 5 along the noon axis. It is worthwhile noting that the dual temperature variation shown in this figure does not entirely stem from exobase conditions. The relatively fast orbital speeds of quasi-satellite atoms can skew the mean-square velocity integral toward a higher net temperature if they comprise an appreciable component of the total density. This causes $T_{\text {kin }}$ for the dual model to be warmer by $\sim 150 \mathrm{~K}$ near $2.0 R_{\mathrm{V}}$ than when satellite atoms are suppressed (refer to Fig. A.3). Kinetic temperatures along the midnight axis (not shown) rarely differ by more than $\sim 25 \mathrm{~K}$ from equiradial noon axis values; throughout, the nightside values are warmer due to the slightly stronger presence of satellite atoms.

Regarding the effects of solar ionization, the extent of exospheric density erosion via SI-loss is displayed in Fig. 2 for the dual model. The quasi-satellite component is still much in evidence (Fig. 4) ; in fact, quasisatellite atoms continue to constitute a major density component in the outer regions of the dual uniform 
exobase model, which might seem surprising. The effect on kinetic temperature is minor (Fig. 5), and is mainly to decrease temperatures throughout most of the exosphere via the preferential removal of satellite atoms (refer to Fig. 4), although at radii $r \geqslant 10.0 R_{\mathrm{V}}$ kinetic temperatures are increased slightly because of the removal of slower-moving atoms. Overall, solar ionization reduces the content of these exosphere models, but does not otherwise alter the evaporative structure, as was found to be the case in terrestrial models (Bishop, 1985).

\section{Empirical exobase results}

Variations in exobase density and temperature at Venus completely determine the structure of the exosphere close to the planet. In the models discussed in this section, the main or thermal exosphere arises from the Hedin-Brinton exobase described earlier, with the neglect of upper thermospheric motions. At altitudes near and above $2.0 R_{\mathrm{v}}$, on the other hand, the exosphere takes on a more global character. "Realistic" exobase parameters for modeling a nonuniform hot population are, unfortunately, much more difficult to justify than in the thermal case, and the hot uniform exobase is retained. While overly simple, this is not likely to introduce gross errors since two factors lead to the expectation that the distribution of bound hot hydrogen atoms will be roughly uniform. First, the major nonthermal sources (charge exchange collisions with ionospheric protons and impact with fastmoving oxygen atoms) are not similarly localized. The first of these is effectively restricted to the region of the atomic hydrogen bulge observed by Brinton et al. (1980) (Kumar et al., 1983), while the second is active on the dayside (Rodriguez et al., 1984). Interestingly, while a simple hot exobase approach might be expected to model an ionospheric charge exchange source fairly well, Figs $2 \mathrm{a}, \mathrm{b}$ of Rodriguez et al. (1984) indicate that a uniform hot exobase of $1000 \mathrm{~K}$ can be used to mimic the $\mathrm{O}^{*}$-impact source. Second, degradation of the bound fast atoms generated by these mechanisms occurs mainly via elastic collisions with cold oxygen atoms near the exobase (Rodriguez et al., 1984); in such collisions, little kinetic energy is lost by the impacting hydrogen atoms, so a fast hydrogen atom will undergo a number of collisions (or bounces) before blending in with the thermal population. This capacity for multiple returns to the exobase, along with the differing regions for the activity of nonthermal sources, imply a variation in the density of nonthermal atoms near the exobase that is much weaker than that exhibited by the thermal population, in turn implying an even weaker variation in "hot" atom density away from the exobase.
Density profiles along the noon axis are shown in Fig. 6 for models with the Hedin-Brinton (HB) cold exobase illustrated in Fig. 1 and the uniform hot exobase; normalization is again with respect to the nosatellite analytic model of the Appendix, with the cold population of that model rescaled to the subsolar HBexobase density when normalizing the nonuniform exobase results. [For purposes of comparison, a dual exosphere model without radiation pressure has been constructed that retains the Hedin-Brinton exobase as a boundary for the cold population but which uses Keplerian algebraic relations to follow particle trajectories (see, e.g., Vidal-Madjar and Bertaux, 1972). This model does not contain satellite atoms, nor has an exopause been incorporated. In this and subsequent figures, profiles belonging to this model are identificd by a "KEP" subscript.] Ncar the cxobasc, the relative density is slightly greater than unity in the HB-exobase models because the exobase temperatures near the subsolar region are warmer than the reference temperature $T_{\text {ref }}$ assumed in the normalization model. As the hot population (shared by all the models) comes to dominate, the evaporative dual models necessarily merge, while the Keplerian profile goes to unity. The SI-modified profile is likewise similar to the corresponding curve in Fig. 2. Near the exobase, the exospheric density at midnight exceeds noon values by two orders of magnitude (see Fig. 1), but the tail ratio drops very quickly due to the colder temperatures of the nightside exobase, as displayed in Fig. 7. Near $1.25 R_{\mathrm{v}}$, dayside densities actually exceed nightside values, due to the warmer exobase temperatures of the sunlit hemisphere. Farther out, where the hot population dominates, the tail ratio merges with the uniform exobase ratio of Fig. 3, while of course the Keplerian model ratio goes to unity since the hot population is then spherically symmetric. Since the quasi-satellite atoms in these models effectively all belong to the hot population, the dual satellite fractions of Fig. 4 are not altered by the use of a realistic cold exobase model. On the other hand, kinetic temperature profiles are changed, especially along the midnight axis (Fig. 8). Again, the presence of quasisatellite atoms raises temperatures considerably, but the rapid jump to high values along the midnight axis is due to the steeper fall-off of the cold population density there.

\section{RELEVANCE TO VENUS AND PAST WORK}

Within the context of evaporative "dual" models patterned after the atomic hydrogen exosphere of Venus, solar radiation pressure causes 


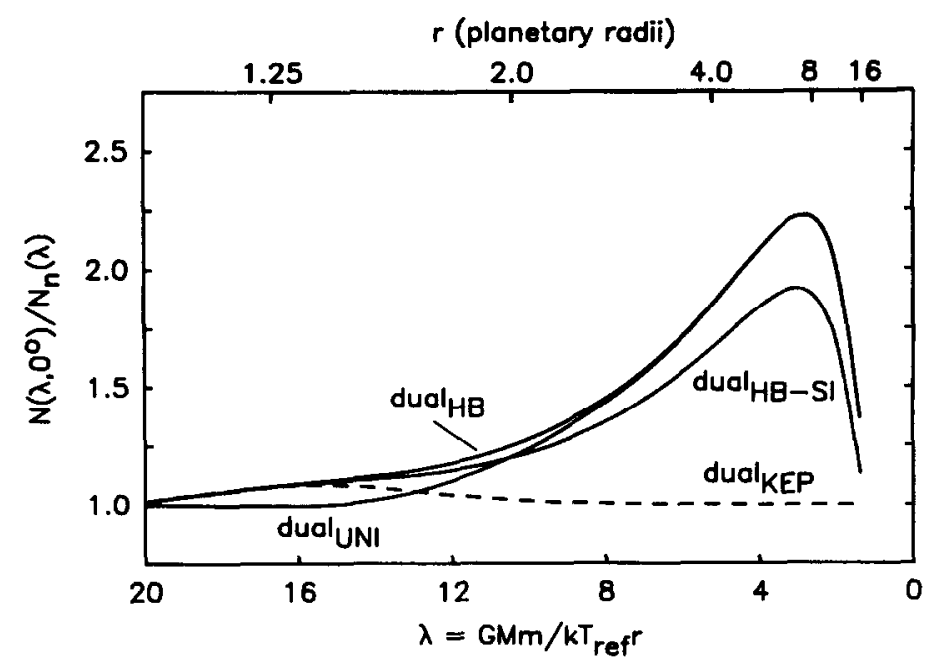

FIG. 6. NORMALIZED DENSITY PROFILES ALONG THE $\chi=0^{\circ}$ AXIS.

Dual evaporative profiles for the cases with radiation pressure and the Hedin-Brinton cold exobase are indicated by the "HB" subscript, with "SI" indicating the inclusion of solar ionization decay. The profile labeled "dual $l_{\text {UNI" }}$ " is from Fig. 2 while that labeled "dual $\left.\right|_{\text {KEP " }}$ comes from the model with the cold HBexobase and particle trajectories given by Keplerian relations.

(i) enhanced densities at outer coronal locations due to the presence of a "hot" quasi-satellite component, this component being non-negligible at $2.0 R_{\mathrm{V}}$ and dominant at radii greater than $4.0 R_{\mathrm{V}}$,

(ii) increased kinetic temperatures (already striking due to the rapid fall-off in cold population density), and

(iii) the imposition of a "tail" of bound atoms, relative to models without radiation pressure. These features can be expected to survive in more realistic treatments. For example, of the reactions invoked in aeronomic studies of the hot corona generation, the charge exchange collision

$$
\mathrm{H}^{+}+\mathrm{H} \rightarrow \mathrm{H}^{*}+\mathrm{H}^{+}
$$

(originating primarily in the early morning bulge region) and the dissociative recombination, momen-

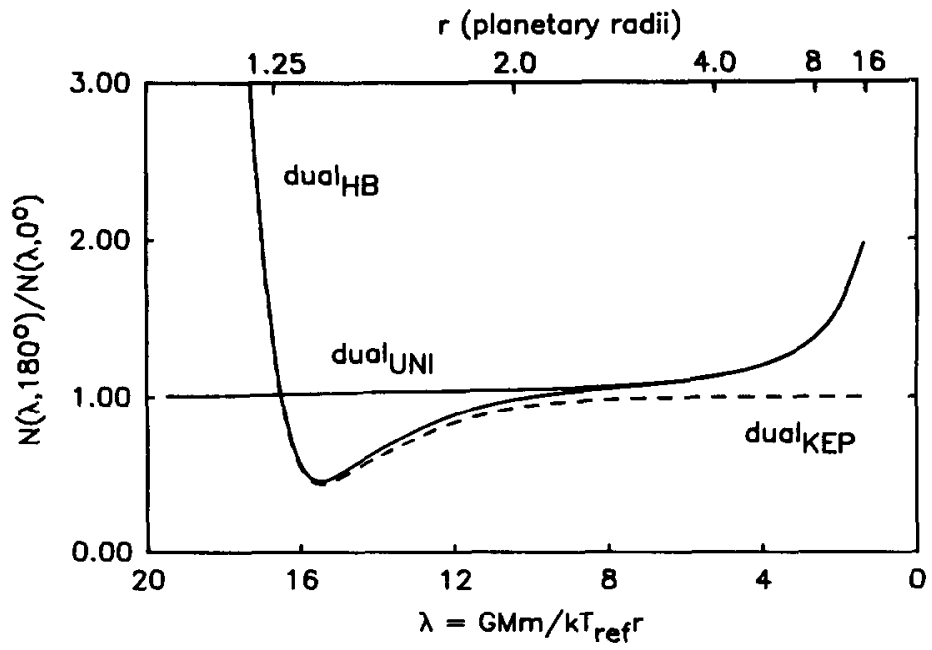

Fig. 7. Tail Ratios with the Hedin-Brinton COLd EXobase.

Labeling scheme is that used in Fig. 6, with the profile "dual ${ }_{\mathrm{UN}}$ " coming from Fig. 3. 


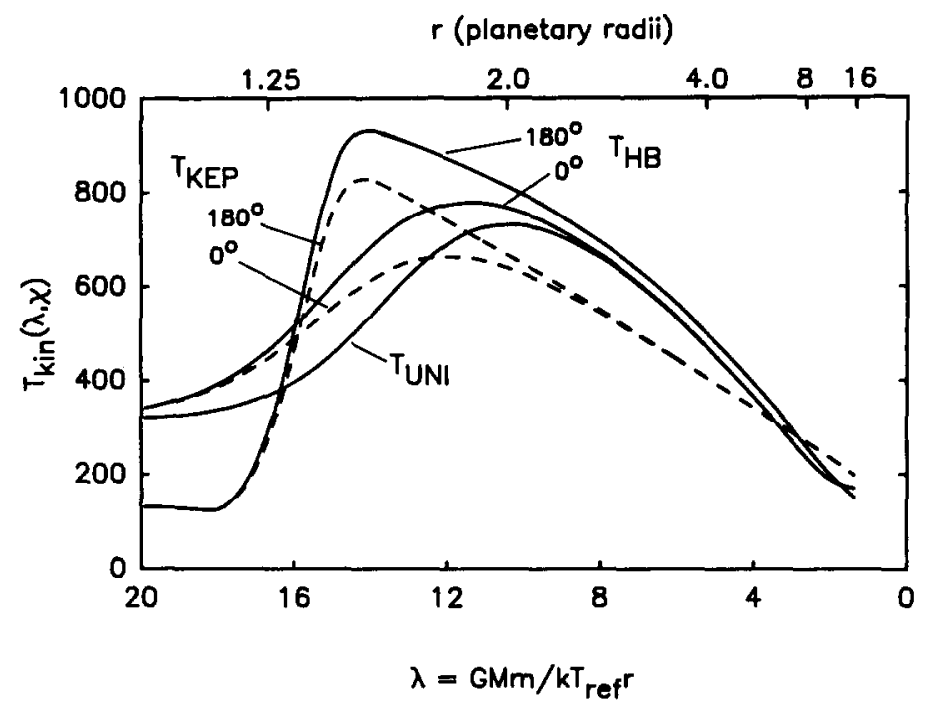

Fig. 8. Kinetic TeMPeratures.

Both noon and midnight axis profiles are shown for those cases using the Hedin-Brinton cold exobase. Labeling scheme is that used in Fig. 6, with the profile " $T_{\text {UNI }}$ " coming from Fig. 5.

tum transfer sequence

$$
\begin{gathered}
\mathrm{O}_{2}^{+}+\mathrm{e}^{-} \rightarrow 2 \mathrm{O}^{*} \\
\mathrm{O}^{*}+\mathrm{H} \rightarrow \mathrm{O}^{*}+\mathrm{H}^{*}
\end{gathered}
$$

are the main channels leading to a hot hydrogen atom $\left(\mathrm{H}^{*}\right)$ flux into the Venus exosphere (Kumar et al., 1983; Rodriguez et al., 1984; Hodges and Tinsley, 1986). Clearly, the kinetics and lateral variations of these reactions and the subsequent collisional thermalization of $\mathrm{H}^{*}$ via elastic scattering off therma] atomic oxygen atoms must be treated in detail to accurately predict densities out in the exosphere; these represent formidable kinetic problems. On the basis of Fig. 6, however, such predictions can be in error by roughly a factor of two if quasi-satellite motions are ignored.

In addition to the purely exospheric features enumerated above and illustrated in Figs 6-8, there are a couple of ways by which radiation pressure can affect the transport of atomic hydrogen at Venus. It has already been noted that the main effect of solar ionization is to reduce the density, particularly in the quasi-satellite component. Most of this loss occurs outside the ionopause and hence is lost to interplanetary space. This "escape" flux is given by

$$
\mathscr{F}_{\mathrm{SI}}=\left(\frac{\lambda_{\mathrm{c}}}{\lambda}\right)^{2} \iiint_{\mathrm{BOUND}} f_{\mathrm{c}} v_{\mathrm{c}} \mu_{\mathrm{c}}\left(1-\mathrm{e}^{-\tau_{\mathrm{F}} / \tau_{\mathrm{s}}}\right) \mathrm{d}^{3} v
$$

where $\tau_{\mathrm{F}}$ is the total flight time of the integration trajectory above the ionopause, the geometric factor references the flux to the cxobasc, and the integration is restricted to the ascending portion of the bound component; $\mathscr{F}_{\text {sI }}$ represents the cumulative loss rate of atoms entering the ionosheath at the point $(\lambda, \chi)$. The subsolar SI-loss computed using this expression with the HB-dual model is $2.0 \times 10^{6} \mathrm{~cm}^{-2} \mathrm{~s}^{-1}$ at a point outside a representative ionopause altitude of $650 \mathrm{~km}$. This is a minimum value for the dayside exobase, in that (i) quasi-satellite trajectories are strongly biased toward launch points away from the subsolar region (Bishop and Chamberlain, 1989) and (ii) for the HB-exobase, $T_{\mathrm{c}}$ remains nearly constant ( $\geqslant 300 \mathrm{~K}$ ) over much of the dayside while $N_{\mathrm{c}}$ increases toward the terminator (particularly in the morning sector) to $\sim 10^{5} \mathrm{~cm}^{-3}$. Thus, at intermediate solar angles, the evaluation of equation (9) at a point just outside the ionopause would encompass both greater cold population densities (the increase in ionopause altitude is not enough to offset the larger $N_{\mathrm{c}}$ values except in the vicinity of the terminator) and greater hot atom erosion (the subsolar result contains a negligible quasi-satellite contribution), leading to a larger mean dayside SI-loss. Unfortunately, it is not possible to extract a dayside average from the current results. Still, $2.0 \times 10^{6} \mathrm{~cm}^{-2} \mathrm{~s}^{-1}$ represents a sizeable loss compared with estimates of dayside hydrogen atom escape due to other mechanisms, for instance $4 \times 10^{6} \mathrm{~cm}^{-2}$ 
$\mathrm{s}^{-1}$ for ionospheric charge exchange (Hodges and Tinsley, 1986, Fig. 5) and $\sim 10^{6} \mathrm{~cm}^{-2} \mathrm{~s}^{-1}$ for loss via O*-impact (McElroy et al., 1982; Rodriguez et al., 1984). It is likely to constitute the main escape mechanism on the dayside, in which case it would play a role in determining the net flux of atomic hydrogen in the Venus dayside thermosphere. It might be noted that equation (9) is sensitive to solar conditions, in that it depends on $\tau_{\mathrm{s}}$ in an exponential manner and also varies with exobase temperature in much the same way as the Jean's escape flux. The stated SI-loss rate is based on the estimate for $\tau_{s}$ of Hodges and Tinsley (1981) and it is not hard to imagine a more realistic description of the state of the shocked solar wind outside the ionopause yielding an upward revision. [When estimating the exospheric SI-loss in the conventional manncr-i.c., $N_{\text {col }}\left(r_{\text {ion }}\right) / \tau_{\mathrm{s}}$, wherc $r_{\text {ion }}$ is the ionopause radius-an underestimate will invariably be obtained because of the neglect of spherical geometry. Rectilinear column densities like those presented in Table 1 really do not provide a good measure of the content of an exosphere. Instead, one ought to use a radial "truncated cone" density

$$
N_{\mathrm{col}}^{*}(r)=\int_{r}^{\infty} N\left(r^{\prime}\right)\left(\frac{r^{\prime}}{r}\right)^{2} \mathrm{~d} r^{\prime}
$$

where the cone has unit cross-sectional area at the reference radius $r$. It is also necessary to be careful in selecting a density profile $N\left(r^{\prime}\right)$. For instance, using a barometric profile yields an infinite result. Even the Chamberlain (1963) models yield an infinite result due to the presence of the escape component; in steady state, escape has been going on for an infinite length of time. In the application at hand, the concern is with the bound component possessing the exopause as a lid (refer to the Appendix); in dimensionless notation,

$$
\begin{aligned}
& N_{\mathrm{col}}^{*}(\lambda)=\frac{2 N_{\mathrm{c}}}{\pi^{1 / 2}} \cdot \frac{G M m}{k T_{\mathrm{c}}} \\
& \cdot \mathrm{e}^{-\lambda} \int_{\lambda_{\mathrm{p}}}^{\lambda} \frac{\mathrm{e}^{\lambda^{\prime}}}{\lambda^{\prime 2}} \gamma\left(\frac{3}{2}, \lambda^{\prime}-\lambda_{\mathrm{p}}\right)\left(\frac{\lambda_{\mathrm{c}}}{\lambda^{\prime}}\right)^{2} \mathrm{~d} \lambda^{\prime} .
\end{aligned}
$$

form hot exobase) for an SI-loss of $0.72 \times 10^{6} \mathrm{~cm}^{-2}$ $\mathrm{s}^{-1}$, severely underestimating the contribution by the hot population.]

The question of thermospheric fluxes is a complicated one, wherein ballistic transport and the upper thermospheric wind system figure largely. Winds have not been considered in this study; nevertheless, it is interesting to consider the exospheric fluxes that arise in the present models. Table 2 displays the vertical effusion speeds at the noon and midnight exobase points for several models. The uniform exobase models exhibit a net flux of bound atoms to the nightside driven entirely by radiation pressure. More precisely, it is due to the variation over the exobase of the potential mar $\cos \chi$ : consider the family of bound trajectories of a given energy that intersect the exobase at the subsolar point; morc atoms leave this point along a specific trajectory than arrive by a factor

$$
\exp \left[\frac{2 \alpha}{\lambda_{c}}\left(1-\cos \chi^{\prime}\right)\right]>1
$$

where $\chi^{\prime}$ is the solar angle of the conjugate exobase intersection. The downward effusion speeds at the anti-solar point listed in Table 2 (uniform exobase entries) are overestimates due to the neglect of the planetary shadow. Still, downward fluxes persist above the anti-solar point in the dual case for $\sim 1000$ $\mathrm{km}$, at which height the hot atom escape flux begins to define the flux character. Of course, whether or not this sort of transport is significant at Venus depends on the strength of the more conventional ballistic flux or transport driven by exobase nonuniformities. As the HB-entries in Table 2 indicate, the flux induced by radiation pressure is minor and only acts to decrease the night-to-day ballistic transport by $\sim 3 \%$ (noon exobase values; with solar ionization, $\sim 6 \%$ ). This ballistic transport is expected to balance in an average sense the day-to-night transport by thermo-

TABle 2. EFfusion SPEEUS* AT EXoHase

\begin{tabular}{lrrr}
\hline Model & & Cold $\dagger$ & Dual $\$$ \\
\hline Uniform : & $0^{\circ}$ & 74 & 101 \\
& $180^{\circ}$ & -72 & -53 \\
HB : & $0^{\circ}$ & -3030 & -2850 \\
& $180^{\circ}$ & 1440 & 1440 \\
HB-SI : & $0^{\circ}$ & -2970 & -2770 \\
& $180^{\circ}$ & 1460 & 1460 \\
HB-KEP : $0^{\circ}$ & -3130 & -2970 \\
& $180^{\circ}$ & 1450 & 1450
\end{tabular}

* In units of $\mathrm{cm} \mathrm{s}^{-1}$.

$\uparrow$ Bound component alone.

$\ddagger$ Escape component included.
Evaluated for a uniform exobase with $N_{c}, T_{c}$ values of $3.2 \times 10^{4} \mathrm{~cm}^{-3}$ and $320 \mathrm{~K}$, respectively (HB-exobase subsolar values), above an ionopause altitude of 650 the hot uniform exobase is $0.73 \times 10^{12} \mathrm{~cm}^{-2}$. Taken together, an SI-loss of $1.7 \times 10^{6} \mathrm{~cm}^{-2} \mathrm{~s}^{-1}$ is indicated for the adopled SI-decay time of $211 \mathrm{~h}$. If, on the other hand, rectilinear column densities above the ionopause had been used [i.e., equation (2)], the analytic model of the Appendix yields $0.42 \times 10^{12} \mathrm{~cm}^{-2}$ (subsolar HB-exobase) and $0.13 \times 10^{12} \mathrm{~cm}^{-2}$ (uni$\mathrm{km}$ yields $N_{\mathrm{col}}^{*}=0.55 \times 10^{12} \mathrm{~cm}^{-2}$ while the result for 
spheric winds that leads to the "pile-up" of atomic hydrogen in the pre-dawn bulge.

Past work on understanding various aspects of the Venus exosphere has led to the models of Hodges and Tinsley $(1981,1986)$ and of Rodriguez et al. (1984). The kinetic study by Rodriguez et al. parallels the approach taken here and developed in Bishop (1985) and Bishop and Chamberlain (1987), by evaluating the exospheric kinetic distribution function along the trajectories executed by hydrogen atoms. Rodriguez et al. went far beyond the aims of the present study in addressing the difficult kinetic problems associated with the Venus exosphere. Their main concerns were in giving a careful description of $\mathrm{O}^{*}$-impact kinetics [equation (8)] using Pioneer Venus measurements to constrain the rate of $\mathrm{O}_{2}^{+}$dissociative recombination [equation (7)] and in assessing the strength and lateral variation of this $\mathrm{H}^{*}$ source relative to the charge exchange source [equation (6)], also prescribed by Pioneer Venus results. However, Rodriguez et al. suggested radiation pressure to be a minor perturbation for locations within $8.0 R_{\mathrm{V}}$ and consequently ignored it, thus failing to realize that the hot population is largely composed of satellite atoms as close in as $4.0 R_{\mathrm{v}}$. Also, their formalism ignores solar wind ionization and photoionization. More importantly, their use of a "column equilibrium" analysis (i.e., that the ballistic transport remains locally controlled and balanced at the exobase) is not appropriate in an exospheric situation and must be relaxed to address the diurnal variation in a consistent manner, in that a net flux is required by the existence of exobase nonuniformities and the upper thermospheric wind system. It should be noted that these limitations are not inherent to a kinetic approach, although the calculations do become more involved as "realism" is approached.

Hodges and Tinsley $(1981,1986)$ present model Venus exospheres obtained by Monte Carlo simulation that incorporate the upper thermospheric and ionospheric conditions revealed by the Pioneer Venus mission in addition to radiation pressure and solar ionization. The simulations reported to date have been confined to exospheric locations inside $2.0 R_{\mathrm{V}}$ and to the consideration of ionospheric charge exchange collisions as the sole source of nonthermal hydrogen. Hodges and Tinsley have convincingly illustrated the non-Maxwellian kinetic distributions resulting from such collisions and have used these to estimate number densities, bulk flow speeds, etc. It is noteworthy that these simulations yield roughly uniform density contours near $2.0 R_{\mathrm{V}}$ in spite of the localization of the hot $\mathrm{H}$ source to the pre-dawn "fountain". At present, such simulations are only capable of providing spatially averaged quantities; these simulations com- plement kinetic studies, the latter being geared more to the analysis of the physics underlying exospheric phenomena.

No attempt has been made in this paper to compare modeled quantities with observations. The commonly cited results of the Mariner 5 (Anderson, 1976) and Mariner 10 (Takacs et al., 1980) flybys have been tacitly acknowledged in the choices of exobase parameter values, so these models will replicate observed behavior near the planet as well as the Chamberlain models initially fitted to the photometer data. Measurements relating to the outer corona are sparser. There is also considerable difficulty in directly comparing calculations and observations in view of the range of uncertainty surrounding various inputs; at this stage of modeling, it is more appropriate to cstablish a basis for theorctical investigation broad enough to encompass various inputs as these become better specified. One observational result will be addressed, however. The hot temperature zone near $2.0 R_{\mathrm{V}}$, illustrated in Figs 8 and A.3, is suggestive of the enhanced "hot" reduction factors seen by Bertaux et al. (1978) and interpreted by them as indicating the position of the ionopause. It was their belief that the rise in temperature indicated by the rapidly varying reduction factors in the altitude range $3000-4000 \mathrm{~km}$ could not be due to a simple superposition of classical exospheres. Instead, the idea was advanced that solar wind ions were being converted to nonthermal neutral atoms, with those on (Keplerian) satellite orbits surviving to accumulate to observable levels with the disappearance of magnetic control, particularly when such orbits are partially protected by the ionopause from subsequent solar wind erosion. In view of the evolution of orbits imposed by radiation pressure, this picture [further advocated in Bertaux et al. (1982)] cannot continue to be upheld, although charge exchange collisions within the solar wind will act to appreciably alter the kinetic distribution on trajectories trespassing the ionopause. Note that while the Bertaux et al. (1978) picture restricts the zone of temperature enhancement to sunlit locations along the ionopause, the expectation here is that this zone extends throughout the nightside as well.

The simple collisionless models advanced in this paper are intended to help assess the importance of radiation pressure dynamics within the Venus exosphere. The acceleration from solar photon scattering is stronger at Venus than at Earth, yet the colder main exobase temperatures result in smaller densities at those altitudes where cumulative effects would be apparent (namely, outside $2.0 R_{\mathrm{v}}$ ). Thus modifications to the atomic hydrogen density profile may not be directly observable; one may hope, however, that con- 
tinued analysis of Pioneer Venus measurements will probe the Venus exosphere more thoroughly than permitted by a flyby mission (see, e.g., Paxton et al., 1988). Radiation pressure can still play an important role, though, through the increased loss of hydrogen from the planet by solar ionization erosion of the quasi-satellite component. The technique used to construct these models is not limited to the simple cases presented, but can incorporate nonuniform in situ processes, as in Bishop and Chamberlain (1987) or Rodriguez et al. (1984). As already noted with the Rodriguez et al. model, the kinetics of the Venus exosphere is difficult to formulate, primarily due to its "nonlocal" character; within the context of exospheric studies in general, though, this is the main point to be addressed. In summary, the emphasis in this paper has been to illustrate the structure imposed by the force fields and simple representatives of the boundary conditions to which exospheric atomic hydrogen at Venus is subject, by assuming a particularly simple exobase kinetic distribution; future work will address the kinetics within the framework of those force fields.

Acknowledgements - The author gratefully acknowledges the support and encouragement of J. W. Chamberlain and the valuable comments and suggestions of the referees. This work was supported by the Atmospheric Research Section, National Science Foundation, under grant ATM-8415118 and by the National Aeronautics and Space Administration under grant NSG-7043, both to Rice University.

\section{REFERENCES}

Anderson, D. E., Jr. (1976) The Mariner 5 ultraviolet photometer experiment: analysis of hydrogen Lyman alpha data. J. geophys. Res. 81, 1213.

Bertaux, J. L. (1978) Interpretation of OGO-5 line shape measurements of Lyman-alpha emission from terrestrial exospheric hydrogen. Planet. Space Sci. 26, 431.

Bertaux, J. L. and Blamont, J. E. (1973) Interpretation of $O G O-5$ Lyman alpha measurements in the upper geocorona. J. geophys. Res. 78, 80.

Bertaux, J. L., Blamont, J., Marcelin, M., Kurt, V. G., Romanova, N. N. and Smirnov, A. S. (1978) Lymanalpha observations of Venera-9 and 10-I. The non-thermal hydrogen population in the exosphere of Venus. Planet. Space Sci. 26, 817.

Bertaux, J. L., Lepine, V. M., Kurt, V. G. and Smirnov, A. S. (1982) Altitude profile of $\mathrm{H}$ in the atmosphere of Venus from Lyman-alpha observations of Venera 11 and Venera 12 and origin of the hot exospheric component. Icarus 52, 221

Bishop, J. (1985) Geocoronal structure: the effects of solar radiation pressure and the plasmasphere interaction. $J$. geophys. Res. 90, 5235.

Bishop, I. and Chamberlain, I. W. (1987) Gencoronal structure 2. Inclusion of a magnetic dipolar plasmasphere. $J$. geophys. Res. 92, 12377.
Bishop, J. and Chamberlain, J. W. (1989) Radiation pressure dynamics in planetary exospheres: a "natural" approach. Icarus (in press).

Brinton, H. C., Taylor, H. A., Jr., Niemann, H. B., Mayr, H. G., Nagy, A. F., Cravens, T. E. and Strobel, D. F. (1980) Venus nighttime hydrogen bulge. Geophys. Res. Lett. 7,865 .

Chamberlain, J. W. (1963) Planetary coronae and atmospheric evaporation. Planet. Space Sci. 11, 901.

Chamberlain, J. W. (1977) Charge exchange in a planetary exosphere: its effect on the distribution and escape of hydrogen. J. geophys. Res. 82, 1.

Chamberlain, J. W. (1979) Depletion of satellite atoms in a collisionless exosphere by radiation pressure. Icarus 39, 286.

Chamberlain, J. W. and Hunten, D. M. (1987) Theory of Planetary Atmospheres. Academic Press, New York.

Cravens, T. E., Gombosi, T. I. and Nagy, A. F. (1980) Hot hydrogen in the exosphere of Venus. Nature 283, 178.

Hedin, A. E., Niemann, H. B., Kasprzak, W. T. and Seiff, A. (1983) Global empirical model of the Venus thermosphere. J. geophys. Res. 88, 73.

Hodges, R. R., Jr. and Tinsley, B. A. (1981) Charge exchange in the Venus ionosphere as the source of the hot exospheric hydrogen. J. geophys. Res. 86, 7649.

Hodges, R. R., Jr. and Tinsley, B. A. (1986) The influence of charge exchange on the velocity distribution of hydrogen in the Venus exosphere. J. geophys. Res. 91, 13649.

Kumar, S., Hunten, D. M. and Pollack, J. B. (1983) Nonthermal escape of hydrogen and deuterium from Venus and implications for loss of water. Icarus 55, 369 .

McElroy, M. B., Prather, M. J. and Rodriguez, J. M. (1982) Escape of hydrogen from Venus. Science 215, 1614.

Niemann, H. B., Kasprzak, W. T., Hedin, A. E., Hunten, D. M. and Spencer, N. W. (1980) Mass spectroscopic measurements of the neutral gas composition of the thermosphere and exosphere of Venus. J. geophys. Res. 85, 7817.

Paxton, L. J., Anderson, D. E., Jr. and Stewart, A. I. F. (1988) Analysis of Pioneer Venus Orbiter Ultraviolet Spectrometer Lyman- $\alpha$ data from near the subsolar region. $J$. geophys. Res. 93, 1766.

Rairden, R. L., Frank, L. A. and Craven, J. D. (1986) Geocoronal imaging with Dynamics Explorer. J. geophys. Res. 91, 13613.

Rodriguez, J. M., Prather, M. J. and McElroy, M. B. (1984) Hydrogen on Venus: exospheric distribution and escape. Planet. Space Sci. 32, 1235.

Takacs, P. Z., Broadfoot, A. L., Smith, G. R. and Kumar, S. (1980) Mariner 10 observations of hydrogen Lyman alpha emission from the Venus exosphere: evidence of complex structure. Planet. Space Sci. 28, 687.

Thomas, G. E. and Bohlin, R. C. (1972) Lyman alpha measurements of neutral hydrogen in the outer geocorona and in interplanetary space. J. geophys. Res. 77, 2752.

Vidal-Madjar, A. and Bertaux, J. L. (1972) A calculated hydrogen distribution in the exosphere. Planet. Space Sci. 20, 1147.

\section{APPENDIX : MODIFIED CHAMBERLAIN MODELS}

The reference models used in this paper are based on the analytic approach of Chamberlain (1963), modified to incorporate an exopause. The modification essentially involves a new approach to the handling of satellite atoms. In the older 


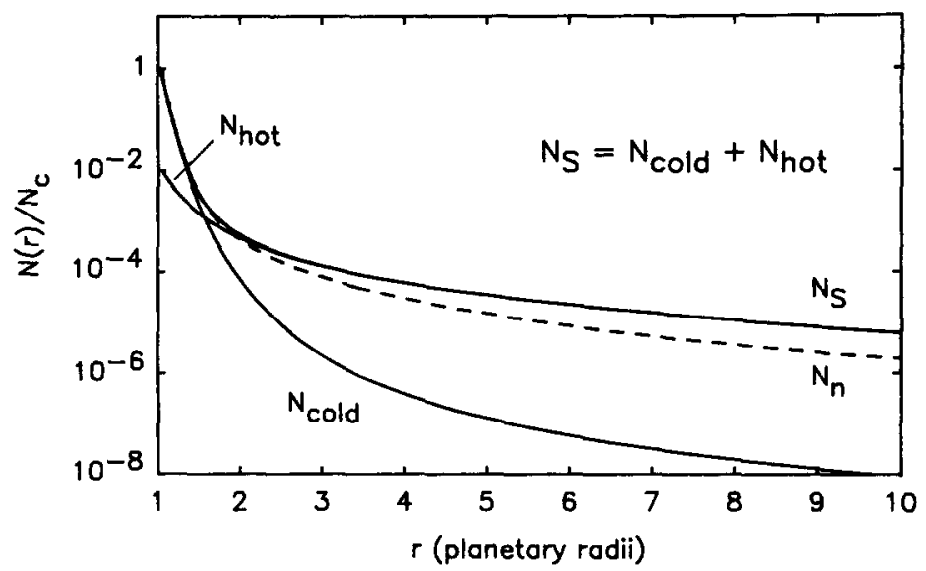

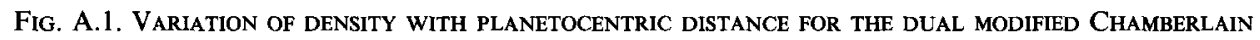
MODEL (CURVE $N_{\mathrm{S}}$ ).

The thermal or "cold" component dominates near the planet while the "hot" population dominates elsewhere. These profiles include an effectively complete satellite component out to the exopause. $N_{n}(\lambda)$ is the dual analytic model density without satellites used for normalization in Figs 1 and 6.

theory, the satellite critical radius $r_{\mathrm{cs}}$ represents a picture of Keplerian satellite atoms generated by (rare) collisions between exospheric constituents to the point where equilibrium is attained with the ballistic component, with an external mechanism (e.g., photoionization) acting to remove satellite atoms with perigee radii above $r_{\mathrm{cs}}$. Here, an exopause radius $r_{\mathrm{p}}$ is introduced as an outer boundary for bound atoms; atoms trespassing this shell are considered to have escaped. Those on trajectories remaining entirely inside the exopause are pictured as (eventually) intersecting the exobase and so are populated according to the assumed exobase kinetic distribution. Thus in the modified version, the satellite critical radius is subsumed into the exopause radius $r_{\mathrm{p}}$. The effective escape relation becomes

$$
\psi^{2}>\lambda-\lambda_{\mathrm{p}}
$$

The partition functions describing the various density components retain the earlier forms with $\lambda \rightarrow \lambda-\lambda_{\mathbf{p}}$ in the evaluation of the incomplete gamma function; for example, the thermal ballistic component partition function becomes (refer to Chamberlain and Hunten, 1987, equation (7.1.34))

$$
\begin{aligned}
\zeta_{\mathrm{bal}}(\lambda)=\frac{2}{\pi^{1 / 2}} & \gamma\left(3 / 2, \lambda-\lambda_{\mathrm{p}}\right)-\frac{2}{\pi^{1 / 2}} \\
& \times\left(1-\lambda^{2} / \lambda_{\mathrm{c}}^{2}\right)^{1 / 2} \mathrm{e}^{-\psi_{1}^{2}} \gamma\left(3 / 2, \lambda-\lambda_{\mathrm{p}}-\psi_{1}^{2}\right)
\end{aligned}
$$

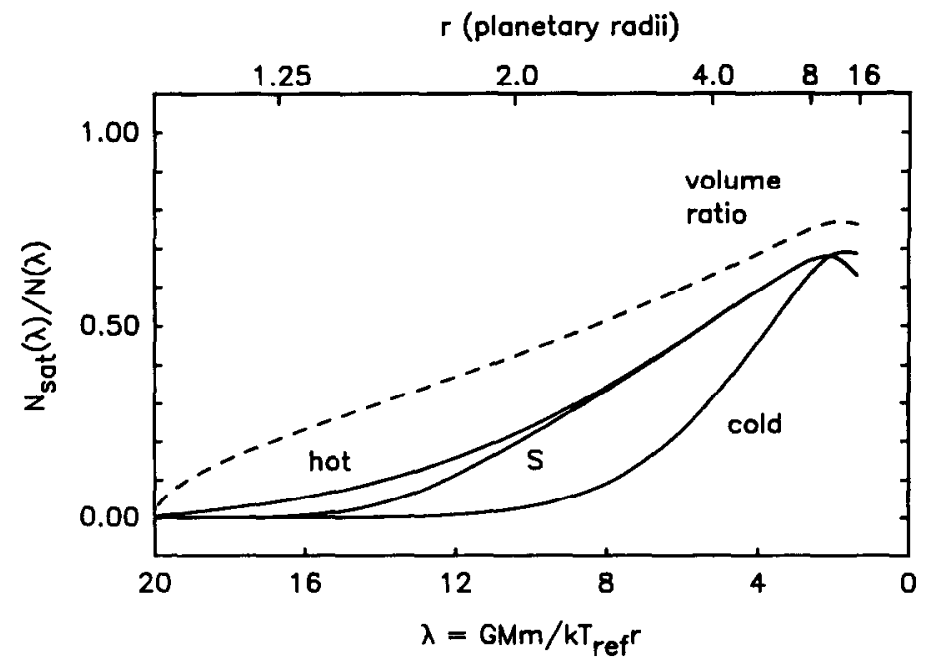

Fig. A.2. SATEllite atom FraCtTonal DENSITY.

The satellite population of the dual analytic exosphere (curve $S$ ) is effectively that of the hot component alone. Also shown is the ratio for velocity space volumes. 


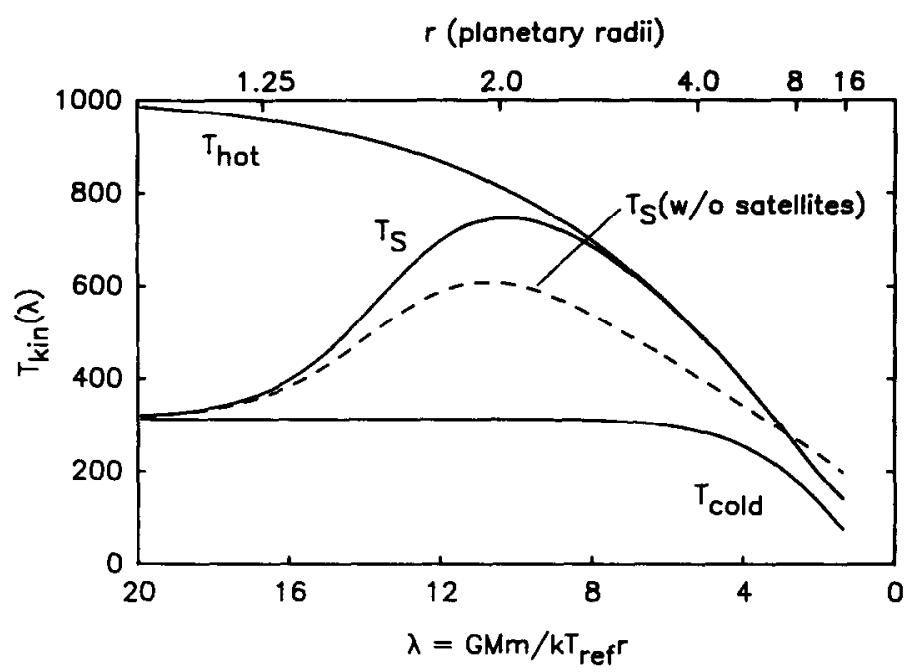

Fig. A.3. Kinftic TFMPFRatuREs.

Refer to equation (A.4) for the defining expression for $T_{\mathrm{S}}$ in terms of $T_{\text {coul }}$ and $T_{\text {hot }}$. Also shown is the dual analytic kinetic temperature with no contribution from satellite atoms.

where $\psi_{1}^{2}=\lambda^{2} /\left(\lambda_{c}+\lambda\right)$ while the Jean's escape flux is [loco cilato, equation (7.1.47)]

$$
\mathscr{F}_{\text {JEANS }}(\lambda)=\frac{N_{\mathrm{c}} U_{\mathrm{c}}}{2 \pi^{1 / 2}} \mathrm{e}^{-\left(\lambda_{\mathrm{c}}-i_{\mathrm{p}}\right)}\left(\lambda_{\mathrm{c}}-\lambda_{\mathrm{p}}+1\right)\left(\frac{\lambda}{\lambda_{\mathrm{c}}}\right)^{2} .
$$

It is no real complication to consider exosphere models arising from "dual" exobase conditions. Densities and escape fluxes simply combine in an additive fashion, while the dual kinetic temperature $T_{\mathrm{S}}$ is given by the rule

$$
\begin{aligned}
T_{\mathrm{S}}(\lambda)= & d_{\text {cold }}^{2} T_{\text {cold }}+d_{\text {hot }}^{2} T_{\text {hot }}+d_{\text {cold }} d_{\text {hot }} \\
& \times\left(T_{\text {cold }}+T_{\text {hot }}\right)+d_{\text {cold }} d_{\text {hot }} \cdot \frac{m}{3 k}\left(\langle v\rangle_{\mathrm{c}}-\langle v\rangle_{\mathrm{h}}\right)^{2}
\end{aligned}
$$

where $d_{\text {cold, hot }}=N_{\text {cold, hot }} /\left(N_{\text {cold }}+N_{\text {hot }}\right), m / 3 k=4.0405 \times 10^{-9}$ $\cdot \mathrm{K} \mathrm{s}^{2} \mathrm{~cm}^{-2}$ for hydrogen atoms, and the angular brackets denote averages over the indicated population kinetic distribution. [In equation (A.4), $N_{\text {cold,hot }}(\lambda)$ denotes the density of the indicated population, e.g., $N_{\text {cold }}=N_{c} \exp \left[-\left(\lambda_{c}-\lambda\right)\right]$ $\zeta_{\text {cold }}(\lambda)$ for the cold analytic model. Refer to Chamberlain (1963), equation (49), for the defining expression of kinetic temperature within the analytic framework, bearing in mind that the imposition of an exopause will necessitate alterations similar to those in equation (A.2).]

To illustrate the dual modified Chamberlain models, the fall-off in density with planetocentric distance is shown in Fig. A.1, using the uniform exobase parameters given in the main text. The dominance of the hot component away from the exobase $\left(r>2.0 R_{\mathrm{v}}\right)$ is well known. Figure A.2 displays the variation in the relative population of satellite atoms, demonstrating that satellite atoms comprise the major density component beyond $\sim 4.0 R_{\mathrm{V}}$ in the absence of loss mechanisms. The dominance of the hot component is again apparent. Also shown is the ratio of the velocity space volume for the satellite component taken alone to that for the complete bound component (ballistic + satellite); this serves to point out that a complete satellite component does not necessarily "fill" the available volume. As noted in the text, the shift from thermal to "hot" kinetic character leads to a prominent temperature maximum illustrated in Fig. A.3. The effect of satellite atoms on the temperature profile is also indicated; by removing the relatively fast moving satcllite atoms, the temperature throughout most of the exosphere is diminished. The asymptotic behavior as $\lambda \rightarrow \lambda_{\mathrm{p}}$ is analogous to that exhibited in the Chamberlain theory as $\lambda \rightarrow 0$. 